\title{
Dez anos de cirurgia da endomiocardiofibrose: o que aprendemos?
}

Carlos R. MORAES*, Jorge V. RODRIGUES*, Cláudio A. GOMES*, Lorella MARINUCCI**, Tereza Cristina COELHO*, Cleusa Lopes SANTOS**, Edgar VICTOR*, Ivan CAVALCANTI*

\section{RBCCV}

MORAES, C. R.; RODRIGUES, J. V.; GOMES, C. A.; MARINUCCI, L.; COELHO, T. C.; SANTOS, C. L.; VICTOR, E.; CAVALCANTI, I. - Dez anos de cirurgia da endomiocardiofibrose: o que aprendemos? Rev. Bras. Cir. Cardiovasc., 2(1):42-52, 1987

RESUMO: Nos últimos 10 anos (1977-1987), 53 pacientes com endomiocardiofibrose foram submetidos a decortição endocárdica e substituição das valvas atrioventriculares. Quarenta e dois eram do sexo feminino e 11, do masculino, variando a idade de 11 a 59 anos (média 31). Os pacientes foram divididos em três grupos: Grupo I, incluindo 25 doentes com lesão biventricular; Grupo II, composto de 23 pacientes com lesão isolada do ventrículo direito, e, por fim, Grupo III, formado de 5 enfermos com doença apenas no ventrículo esquerdo. Todos estavam na classe funcional III ou IV, de acordo com a classificação da New York Heart Association. A mortalidade, nos primeiros 30 dias após a cirurgia, foi de $20,7 \%$ (11 casos). Dos 42 sobreviventes, $21(39,6 \%)$ tiveram um pós-operatório imediato bastante tormentoso. Houve $10(18,8 \%)$ óbitos tardios. Uma paciente foi reoperada dois anos depois, para substituição da valva mitral, que havia sido preservada na primeira intervenção. Dentre os 32 sobreviventes (tempo de evolução de 4,1 pacientes/ano), somente $22(41,5 \%)$ estão na classe funcional I ou II. A curva atuarial mostrou que a probabilidade de sobrevida em 5 anos é de $75 \%$. A despeito da elevada mortalidade imediata, ou tardia, e do fato de que menos da metade dos pacientes operados apresentam melhora clínica na evoluçāo a longo prazo, o tratamento cirúrgico é a única esperança de recuperação para pacientes com endomiocardiofibrose. Aspectos técnicos importantes da operação são descritos.

DESCRITORES: endomiocardiofibrose, cirurgia.

\section{INTRODUÇÃO}

A ressecção do endocárdio ventricular, associada à substituição, ou ao reparo, das valvas atrioventriculares, como tratamento cirúrgico da endomiocardiofibrose, introduzido por DUBOST, em 1971 (referido por PRIGENT et alii ${ }^{33}$ ), incluiu, no rol das doenças cardiovasculares passiveis de cor- reção cirúrgica, uma enfermidade até então de interesse somente anátomo-clínico e de grave prognóstico.

Nos últimos anos, um número crescente de publicações tem enriquecido a literatura médica com casos de endomiocardiofibrose submetidos ao tratamento cirúrgico $2-6,8-16,18-35,37,38$. Entretanto,

Trabalho realizado no Centro de Ciências de Saúde da Universidade Federal de Pernambuco e no Serviço de Cirurgia Cardiotorácica (Prof. Carlos R. Moraes) do Real Hospital Português de Beneficência em Pernambuco. Recife, PE, Brasil.

Apresentado ao 14: Congresso Nacional de Cirurgia Cardiaca. Salvador, BA, 27 e 28 de março, 1987.

- Do Centro de Ciências de Saúde da Universidade Federal de Pernambuco e do Serviço de Cirurgia Cardiotorácica (Prof. Carlos R. Moraes) do Real Hospital Português de Beneficência em Pernambuco.

* Do Serviço de Cirurgia Cardiotorácica (Prof. Carlos R. Moraes) do Real Hospital Português de Beneficência em Pernambuco.

Endereço para separatas: Carlos R. Moraes. Av. Bernardo Vieira de Melo, 4405/491. Jaboatāo. 56.100 Recife, PE, Brasil 
MORAES, C. R.; RODRIGUES, J. V.; GOMES, C. A.; MARINUCCI, L.; COELHO, T. C.; SANTOS, C. L.; VICTOR, E.: CAVALCANTI. I. - Dez anos de cirurgia da endomiocardiofibrose: o que aprendemos? Rev. Bras. Cir. Cardiovasc., 2(1):42-52. 1987.

a maioria dos trabalhos relata casos isolados, ou casuísticas pouco numerosas, tornando-se difícil uma avaliação acurada, não só dos problemas envolvidos na cirurgia de tão complexa doença, mas, sobretudo, dos reais benefícios a longo prazo desse tipo de procedimento.

No presente trabalho, procuramos, de maneira bastante objetiva, fazer uma análise crítica da nossa experiência no tratamento cirúrgico da endomiocardiofibrose, iniciada há 10 anos.

\section{CASUÍSTICA E MÉTODOS}

Material clínico - Nos últimos 10 anos (1977-1987), 53 pacientes foram submetidos ao tratamento cirúrgico da endomiocardiofibrose, em nossos Serviços. Quarenta e dois $(79,2 \%)$ eram do sexo feminino e $11(20,8 \%)$, do masculino. A idade variou de 11 a 59 anos ( $\bar{x} 31)$.

Todos os pacientes estavam na fase final da insuficiência cardíaca, sendo que 33 se inseriam na classe funcional III e 20 , na classe IV, de acordo com a classificação da New York Heart Association. O diagnóstico pré-operatório definitivo foi estabelecido por cineangiocardiografia seletiva, evidenciando deformidades ventriculares típicas previamente descritas ${ }^{29}$.

No que concerne à localização da doença, os pacientes foram divididos em três grupos (Tabela 1): Grupo I, composto de $25(47,1 \%)$ doentes apresentando endomiocardiofibrose bilateral; Grupo II, formado por $23(43,5 \%)$ casos com doença isolada do ventrículo direito e, finalmente, Grupo III, constituído por $5(9,4 \%)$ enfermos com lesão apenas do ventrículo esquerdo.

TABELA 1

DIVISATO DOS GRUPOS

\begin{tabular}{cccr}
\hline GRUPO & LOCALIZAÇATO DA DOENCAA & N:CASOS & $\%$ \\
\hline I & Biventricular & 25 & 47,1 \\
II & VD & 23 & 43,5 \\
III & VE & 5 & 9,4 \\
\hline
\end{tabular}

$$
X^{2}=13,736 \quad p<0,01
$$

$\mathrm{VD}=$ ventrículo direito

$\mathrm{VE}=$ ventrículo esquerdo

Técnica cirúrgica - A técnica cirúrgica utilizada já foi previamente descrita em detalhes ${ }^{30}$. Todos os pacientes foram operados através de esterno- tomia mediana, com circulação extracorpórea convencional a $30^{\circ} \mathrm{C}$, após canalização da aorta ascendente e de ambas as veias cavas. A perfusão foi interrompida em 1 paciente e ambas as cânulas venosas tiveram de ser trocadas, já que foram bloqueadas por trombos existentes no átrio direito. Realizou-se proteção miocárdica por infusão de solução cardioplégica gelada na raíz da aorta, associada à hipotermia tópica do coração.

A endocardiectomia direita (Tabela 2 ) foi realizada em 48 pacientes, dos quais 25 tinham lesão biventricular (Grupo I), e 23 apresentavam lesão isolada do ventrículo direito (Grupo II). Em todos, a via de acesso foi uma ampla atriotomia direita. Quinze doentes $(31,2 \%)$ tinham trombos no átrio direito e $1(2 \%)$, no ventrículo direito. Cinco pacientes $(10,4 \%)$, todos do Grupo I, mostravam uma área de fibrose limitada à ponta do ventrículo direito. Nestes casos, procedeu-se à endocardiectomia, preservando-se, contudo, a valva tricúspide. Nos demais, a valva tricúspide foi substituída por uma prótese.

\section{TABELA 2}

ENDOCARDIECTOMIA DIREITA
48 Casos

A endocardiectomia esquerda (Tabela 3 ) foi realizada em 30 pacientes, dos quais 25 tinham lesão bilateral (Grupo I) e 5 apresentavam lesão isolada do ventrículo esquerdo (Grupo III). A via de acesso ao ventrículo esquerdo variou de acordo com o tamanho do átrio esquerdo e com o grau de regurgitação mitral. Em 15 casos (50\%), utilizou-se uma atriotomia esquerda. Em todos eles, o átrio esquerdo estava aumentado, incluindo-se os 5 pacientes do Grupo III e 10 do Grupo I. Em 9 doentes $(30 \%)$, efetuou-se pequena ventriculotomia esquerda. Essa incisão foi feita eletivamente, apenas nos casos em que a cineventriculografia mostrava fibrose limitada à ponta do ventrículo esquerdo e discreta insuficiência mitral. Finalmente, em 6 doentes $(20 \%)$, o ventrículo esquerdo foi abordado por uma incisão no septo interatrial. Trombos no ventrículo esquerdo foram encontrados em 3 casos (10\%). Em 7 pacientes $(23,3 \%)$, a valva mitral foi preservada. Nos outros 23 $(76,7 \%)$, ela foi substituída por uma prótese. 
MORAES, C. R.; RODRIGUES, J. V.; GOMES, C. A.; MARINUCCI, L.; COELHO, T. C.; SANTOS, C. L.; VICTOR, E.; CAVALCANTI.

I. - Dez anos de cirurgia da endomiocardiofibrose: o que aprendemos? Rev. Bras. Cir. Cardiovasc., 2(1):42-52. 1987.

TABELA 3

ENDOCARDIECTOMIA ESQUERDA
30 Casos

Nos 53 pacientes operados, foram realizadas 66 substituições valvares. Somente em dois casos empregaram-se próteses mecânicas tipo Medtronic-Hall. Nos demais, utilizaram-se 64 biopróteses: 33 de pericárdio bovino; 19 de dura-máter e, por fim, 12 válvulas de porco. Nos últimos 10 casos, usaram-se biopróteses de baixo perfil, com o intuito de evitar obstrução da via de saída dos ventrículos e arritmias.

Métodos de estatística - Foram calculadas as médias e os desvios padrões. No sentido de identificar possíveis diferenças entre os três grupos de doentes, foram aplicadas as provas de $\mathrm{FI}$ SHER $^{36}$, Qui-quadrado ${ }^{36}$, binominal ${ }^{36}$, veromáxima semelhança com o fator de correção de Williams ${ }^{1}$, aceitando-se como nível de significação estatística, os valores correspondentes a 0,05 .

Para o estabelecimento da curva aturial e para o cálculo do tempo pessoa/período de observação de todos os sobreviventes e dos sobreviventes pertencentes aos Grupos I, II e III, foi empregado o método da tábua de vida ${ }^{17}$.

Finalmente, para estudar-se a distribuição pré e pós-operatória dos doentes sobreviventes nos tipos funcionais da classificação da New York Heart Association, foi empregado um modelo linear, segundo as especificações recomendadas por CORDEIRO?.

\section{RESULTADOS}

Mortalidade imediata - A mortalidade nos 30 primeiros dias após a cirurgia foi de $20,7 \%$ (Tabela 4). Onze pacientes faleceram, em decorrência de: síndrome de baixo débito ( 6 casos), arritmia ( $1 \mathrm{ca}$ so), acidente vascular cerebral (1 caso), insuficiência renal aguda ( 1 caso), embolia pulmonar ( 1 caso) e sepsis (1 caso). Não houve diferença estatisticamente significante ( $p=0,274)$ entre causas de morte cardíacas ( 7 casos) e não cardíacas (4 casos).
TABELA 4

\begin{tabular}{l}
\hline $\begin{array}{c}\text { MORTALIDADE IMEDIATA } \\
11 \text { CaSOS }(20,7 \%)\end{array}$ \\
\hline CAUSAS \\
\hline $\begin{array}{l}\text { Sindrome de baixo débito (6) } \\
\text { Arritmia } \\
\text { Acidente vascular cerebral } \\
\text { Insuficiência renal aguda } \\
\text { Embolia pulmonar } \\
\text { Sepsis }\end{array}$ \\
\hline $\mathrm{p}=0,274$
\end{tabular}

A mortalidade foi diferente nos três grupos (Tabela 5), sendo de $28 \%$ no Grupo I, composto de 25 pacientes com lesão biventricular, e de 17,3\% no Grupo II, formado por 23 doentes com lesão isolada do ventrículo direito. Não houve débitos entre os 5 casos do Grupo III que apresentavam doença apenas do ventrículo esquerdo. Essa diferença, contudo, não foi estatisticamente significativa ( $p$ > $0,05)$.

TABELA 5

RELAÇÃO MORTALIDADE IMEDIATAVGRUPO

\begin{tabular}{cccc}
\hline GRUPO & N.CASOS & OBITOS & $\%$ \\
\hline I & 25 & 7 & 28,8 \\
II & 23 & 4 & 17,4 \\
III & 5 & - & - \\
\hline
\end{tabular}

$$
G^{2}=1,498 \quad p>0,05
$$

Complicaçôes pós-operatórias - Dos 42 pacientes que sobreviveram à cirurgia, $21(39,6 \%)$ apresentaram um total de 36 complicações graves, mas não fatais (Tabela 6). Observa-se que ocorreram 26 complicações cardíacas e 10 extracardiacas, tornando-se este fato estatisticamente significante $(p<0,01)$.

TABELA 6

COMPLICAÇÓES PÓS-OPERATÓRIAS NĀO FATAIS 21 Casos $(39,6 \%)$

$\begin{array}{lr}\text { Cardiacas } & \\ \text { Síndrome de baixo débito } & 12 \\ \text { BAVT } & 7 \\ \text { Arritmias } & 7 \\ \text { Não Cardíacas } & \\ \text { Insuficiência renal } & 3 \\ \text { Hemorragia } & 3 \\ \text { Infecção } & 3 \\ \text { Embolia pulmonar } & 1 \\ \quad \text { TOTAL } & 36\end{array}$

$$
X^{2}=7,111 \quad p<0,01
$$


A incidência de complicaçōes entre os sobreviventes dos Grupos I, II e III (Tabela 7) foi, respectivamente, de $61,1 \%, 42,1 \%$ e $40 \%$, não havendo diferença significativa $(p>0,05)$.

TABELA 7 RELAÇĀO INCIDENCIA DE COMPLICAÇŌES/GRUPO

\begin{tabular}{cccc}
\hline GRUPO & SOBREVIVENTES & COMPLICACYESS & $\%$ \\
\hline I & 18 & 11 & 61,1 \\
II & 19 & 8 & 42,1 \\
III & 5 & 2 & 40,0 \\
\hline
\end{tabular}

$$
G^{2}=0,498 \quad p>0,05
$$

Causas de morte e complicações pós-operatórias - Uma análise conjunta das complicações fatais e não fatais (Tabela 8) demonstrou que a freqüência de complicações cardiacas no pós-operatório é significativamente maior do que a de causa extracardiaca $(p<0,01)$.

TABELA 8

COMPLICAÇÖES PÓS-OPERATÓRIAS Fatais e Não-Fatais

32 Casos $(60,3 \%)$

$\begin{array}{lc}\text { Cardíacas } & \\ \text { Síndrome de baixo débito } & 18(32,9 \%) \\ \text { Arritmia } & 8(15 \%) \\ \text { BAVT } & 7(13,2 \%) \\ \text { Não cardiacas } & \\ \text { Insuficiência renal } & 4(7,5 \%) \\ \text { Infecção } & 4(7,5 \%) \\ \text { Hemorragia } & 3(5,6 \%) \\ \text { Embolia pulmonar } & 2(3,7 \%) \\ \text { Acidente vascular cerebral } & 1(1,8 \%)\end{array}$

$$
X^{2}=7,680 \quad p<0,01
$$

Bloqueio $A-V$ total ocorreu em 7 casos $(13,2 \%)$, dos quais, em $3(5,6 \%)$, foi temporário e, em 4 $(7,5 \%)$, foi definitivo, sendo necessário o implante de marcapasso permanente.

Reoperação - Apenas uma paciente foi reoperada no segundo ano de evolução, para substituição da valva mitral, a qual havia sido preservada na primeira intervenção. Neste caso, em que a paciente viria a falecer no 4 : ano de evolução pós-operatória, não se observou recidiva da fibrose endocárdica.

Mortalidade tardia - A mortalidade tardia (Tabela 9 ) foi de $18,8 \%$. Dez pacientes faleceram, devido a: insuficiência cardiaca ( 3 casos) acidente vascular cerebral ( 2 casos), sepsis ( 2 casos), hepatite
(1 caso), infecção intestinal (1 caso) e embolia pulmonar ( 1 caso). Exceto uma paciente, falecida quatro anos após a cirurgia, todos os outros óbitos ocorreram em períodos de tempo que variaram de 40 dias a 12 meses de pós-operatório.

TABELA 9

MORTALIDADE TARDIA

10 Casos $(18,8 \%)$

\section{CAUSAS}

\begin{tabular}{ll} 
Insuficiência cardiaca & 3 \\
Acidente vascular cerebral & 2 \\
Sepsis & 2 \\
Hepatite & 1 \\
Infecção intestinal & 1 \\
Embolia pulmonar & 1 \\
\hline
\end{tabular}

A relação entre a mortalidade tardia e os sobreviventes dos Grupos I, II e III não se revelou estatisticamente significativa $(p>0,05)$.

TABELA 10

RELACÃO MORTALIDADE TARDIA GRUPO

\begin{tabular}{rccc}
\hline GRUPO & SOBREVIVENTES & N:CASOS & 16.6 \\
\hline I & 18 & 3 & 16,6 \\
II & 19 & 7 & 36,8 \\
III & 5 & - & - \\
\hline
\end{tabular}

$$
\mathrm{G}^{2}=2,807 \quad \mathrm{p}>0,05
$$

Evolução tardia - Trinta e dois pacientes sobrevivem atualmente, e o tempo de evolução é de 4,1 pacientes/ano (variando de 2 a 96 meses). Observa-se, na Figura 1, que 22, ou seja $41,5 \%$ da série total de operados, estão na classe funcional I ou II, de acordo com a classificação da New York Heart Association. Os outros 10 continuam apresentando manifestações clínicas importantes de insuficiência cardíaca. Aplicando-se um modelo linear, verificou-se haver significação estatística nas modificações funcionais pré e pós-operatórias $(p>0,05)$.

TABELA 11

\begin{tabular}{ccc}
\hline $\begin{array}{c}\text { INTERVALO } \\
\text { (ano) }\end{array}$ & $\begin{array}{c}\text { PROBABILIDADE DE } \\
\text { SOBREVIDA (\%) }\end{array}$ & ERRO PADRÃO \\
\hline $1 / 12$ & 95,74 & 1,33 \\
1 & 93,79 & 1,39 \\
2 & 87,32 & 1,51 \\
3 & 81,95 & 2,39 \\
4 & 78,22 & 2,63 \\
5 & 75,32 & 3,01 \\
\hline
\end{tabular}




\section{EVOLUGÃO TARDIA 32 C ASOS \\ 4,1 PACIENTES / ANO $(2-96$ meses )}

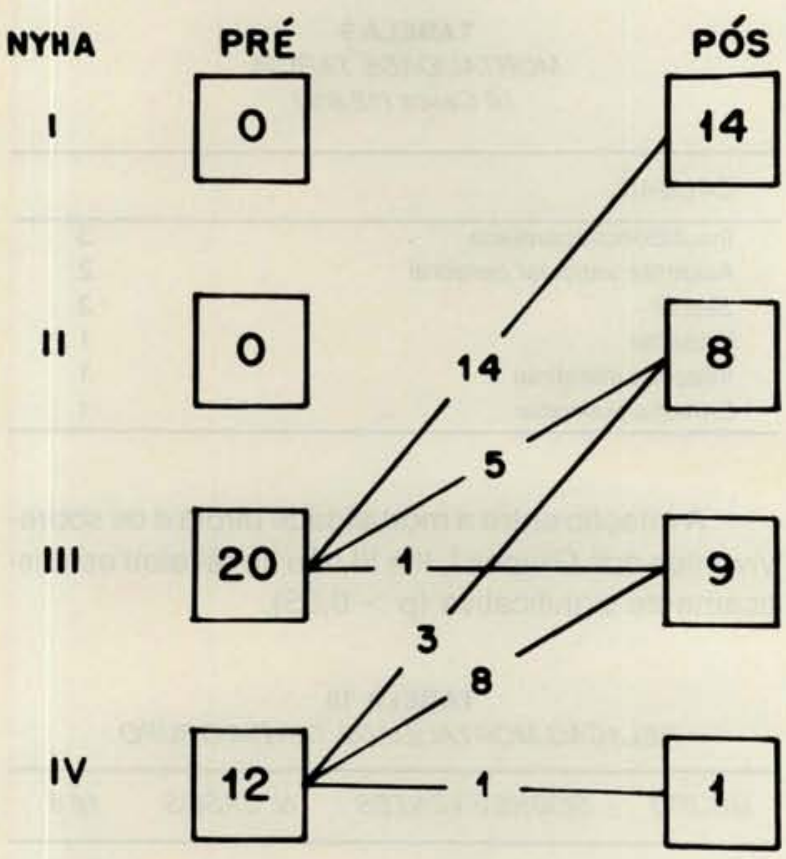

$$
X^{2}=4,042 \quad P<0,05
$$

Fig. 1

Nas Figuras 2, 3 e 4, mostra-se, separadamente, a evolução funcional dos sobreviventes nos Grupos I, II e III. O tempo de evolução, nos três grupos, é pouco diferente, sendo, respectivamente, $3,10,4,2$ e 4,1 pacientes/ano. Também aplicando-se o modelo linear, verificou-se que as modificaçōes funcionais dos doentes, nos Grupos I e II, foram significativas $(p>0,05)$. O referido modelo não pôde ser utilizado no Grupo III, graças ao reduzido número de casos.

Curva atuarial - Na Tabela 9 e na Figura 5 , observam-se as probabilidades de sobrevida e respectivos erros padrões, desde o período pós-operatório imediato até cinco anos depois da cirurgia, com intervalos de um ano. A probabilidade de sobrevivência aos 5 anos de pós-operatório é de $75 \%$.

\section{DISCUSSÃO}

O diagnóstico de endomiocadiofibrose vem sendo feito com uma freqüência cada vez maior, no Brasil, especialmente no Nordeste, possibilitan- do-nos operar 53 pacientes portadores dessa doença, nos últimos 10 anos.

As características clínicas e morfológicas da endomiocardiofibrose estāo, atualmente, bem estabelecidas, apesar de que a sua etiologia permaneça ainda obscura.

Em nossa casuística, ressaltam-se a grande predominância do sexo feminino $(79,2 \%$ ) e a raridade das formas isoladas do ventrículo esquerdo $(9,4 \%)$, existindo uma certa discrepância com outros relatos ${ }^{14,25}$. Pensamos, na ausência de outra explicação, que essa é mais uma das caprichosas diferenças regionais, observadas na apresentação da endomiocardiofibrose.

O grave prognóstico dessa enfermidade, a natureza irreversível da doença e a resposta pouco satisfatória ao tratamento clínico são bem conhecidos $^{2}{ }^{28}$. O tratamento cirúrgico, que consiste na

\section{EVOLUCÃO TARDIA Grupo I 15 CASOS \\ 3.10 PACIENTES / ANO (2- 72 meses)}

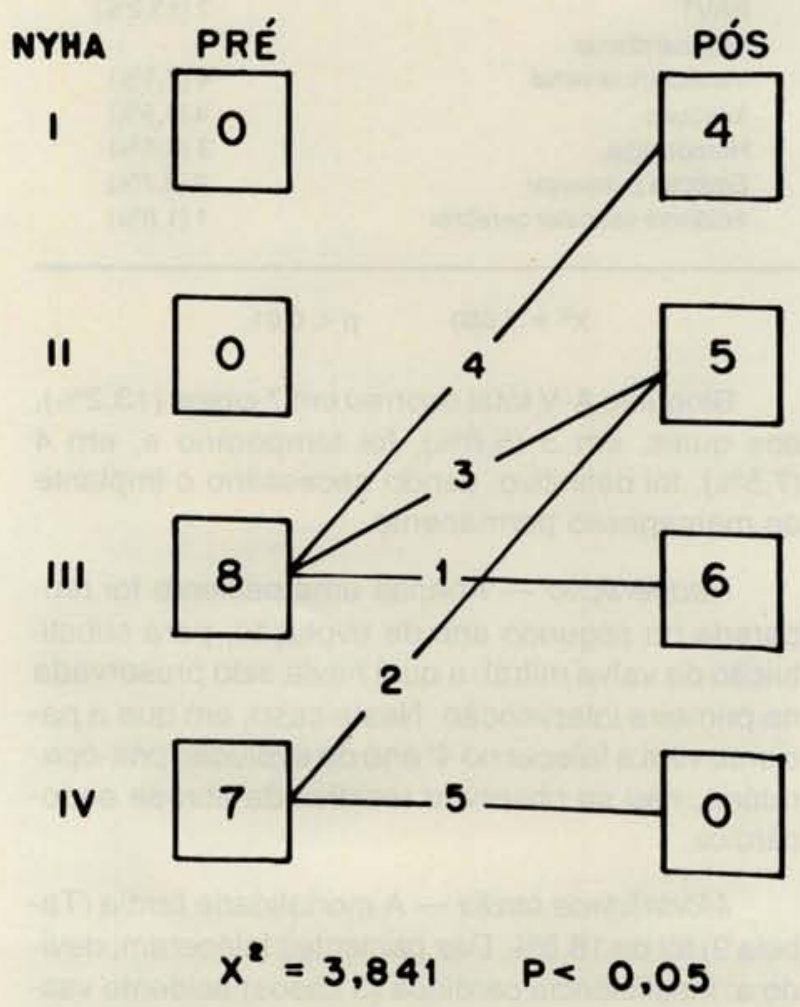

Fig. 2 
ressecção da fibrose endocárdica associada à substituição, ou ao reparo das valvas atrioventriculares, é, atualmente, a única esperança de modificar a desfavorável história natural da endomiocardiofibrose.

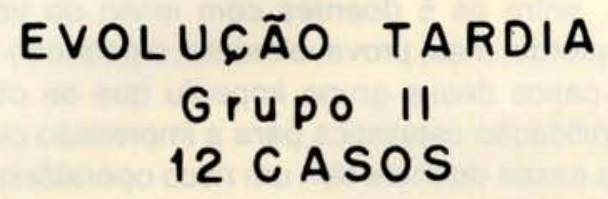

\subsection{PACIENTES / ANO $(7-96$ meses )}

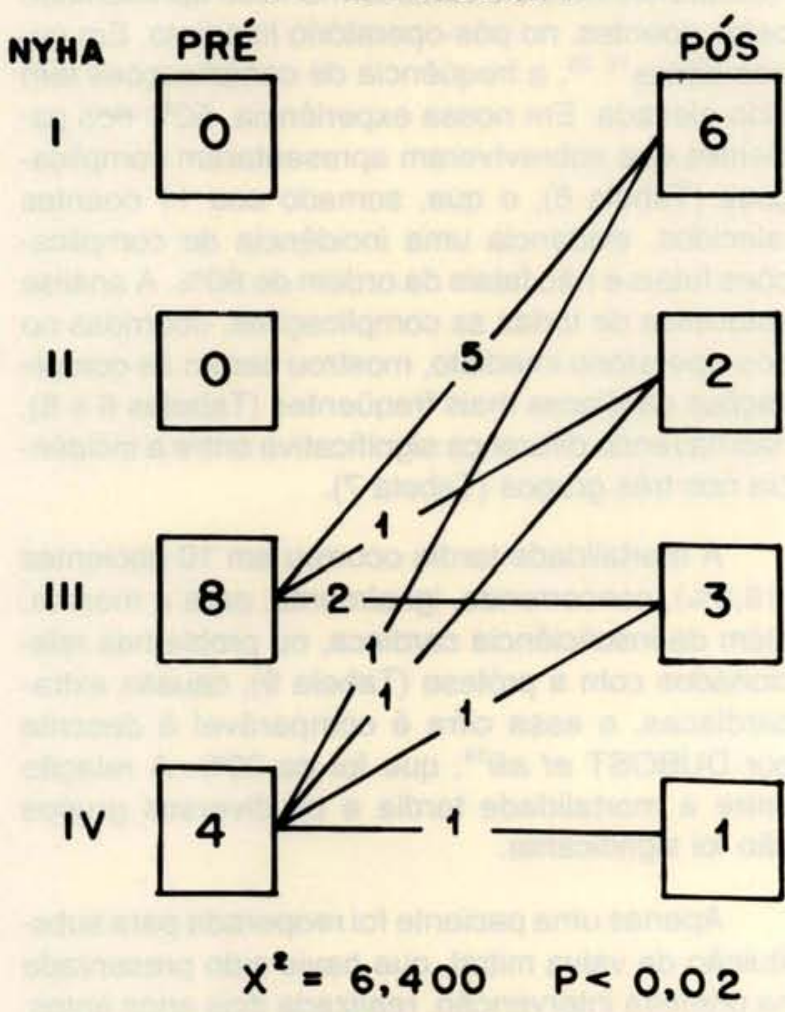

Fig. 3

A técnica cirúrgica é relativamente simples. Durante a endocardiectomia, o cirurgião deve permanecer no lado da fibrose, evitando lesão do miocárdio subjacente. Este procedimento é fácil no ventrículo direito, cujo acesso é sempre obtido por uma incisão no átrio direito, que se encontra sempre muito dilatado. Contudo, a incidência de arritmias (especialmente bloqueio $\mathrm{A}-\mathrm{V}$ total) é elevada após a endocardiectomia direita, em decorrência de traumatismo do sistema de condução. Em nossa série, 7 pacientes $(13,2 \%)$ apresentaram bloqueio A-V total no pós-operatório imediato, sendo que, em 4 deles $(7,5 \%)$, o bloqueio foi definitivo, exigindo o implante de marcapasso. Recentemente, temos seguido a sugestão, feita por MÉTRAS et alii ${ }^{25}$, de deixar uma pequena faixa de fibrose na região do feixe de His, o que parece diminuir a incidência de arritmias no pós-operatório.

A endocardiectomia esquerda também é uma operação fácil, quando o átrio esquerdo é aumentado de tamanho. No entanto, nos casos de doença bilateral, o átrio esquerdo, freqüentemente, é de tamanho normal, exigindo, assim, que o cirurgião aborde o ventrículo esquerdo através de uma incisão no septo interatrial, ou por uma ventriculotomia esquerda. Isto ocorreu em $50 \%$ dos nossos casos, dificultando, evidentemente, a operação. Talvez, uma maneira de se obter um acesso mais fácil ao ventrículo esquerdo seja a realização da operação através de toracotomia anterior direita ${ }^{*}$, se bem que, na eventualidade de perfuração do coração durante a endocardiectomia, a correção da lesão tornar-se-ia muito difícil.

Nossa experiência tèm mostrado que uma pequena incisão na ponta do ventrículo esquerdo constitui-se na via de acesso ideal, nos casos cuja cineventriculografia mostra fibrose daquela cavida-

\section{EVOLUCÃO TARDIA Grupo III 5 CASOS}

\section{4,1 PACIENTES / ANO ( 24-90 meses)}

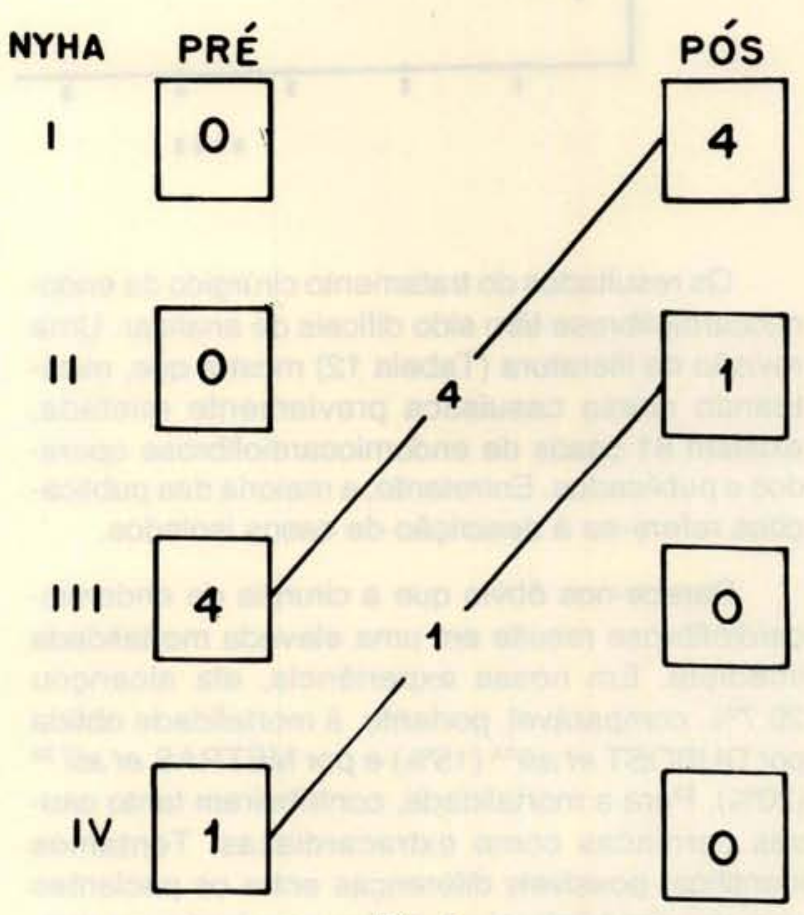

Fig. 4 
MORAES, C. R.; RODRIGUES, J. V.; GOMES, C. A.; MARINUCCI, L.; COELHO, T. C.; SANTOS. C. L.; VICTOR. E.; CAVALCANTI.

I. - Dez anos de cirurgia da endomiocardiofibrose: o que aprendemos? Rev. Bras. Cir. Cardiovasc., 2(1):42-52. 1987.

de com valva mitral competente ou discretamente insuficiente ${ }^{30}$

Um outro aspecto importante de técnica cirúrgica a ser considerado é o tipo de prótese valvular a ser utilizada. $\mathrm{Na}$ endomiocardiofibrose, a cavidade ventricular é pequena e, mesmo após a decorticação, a colocação de uma prótese pode provocar obstrução da via de saída, ou arritmias por compressão do sistema de condução. Isto foi observado em alguns de nossos casos e, por isso, temos, atualmente, utilizado apenas próteses de baixo perfil.

\section{CiruRgIA DA ENDOMIOCARDIOFIBROSE 53 CASOS}

PROBABILIDADE DE SOBREVIDA PÓS-OPERATÓRIA

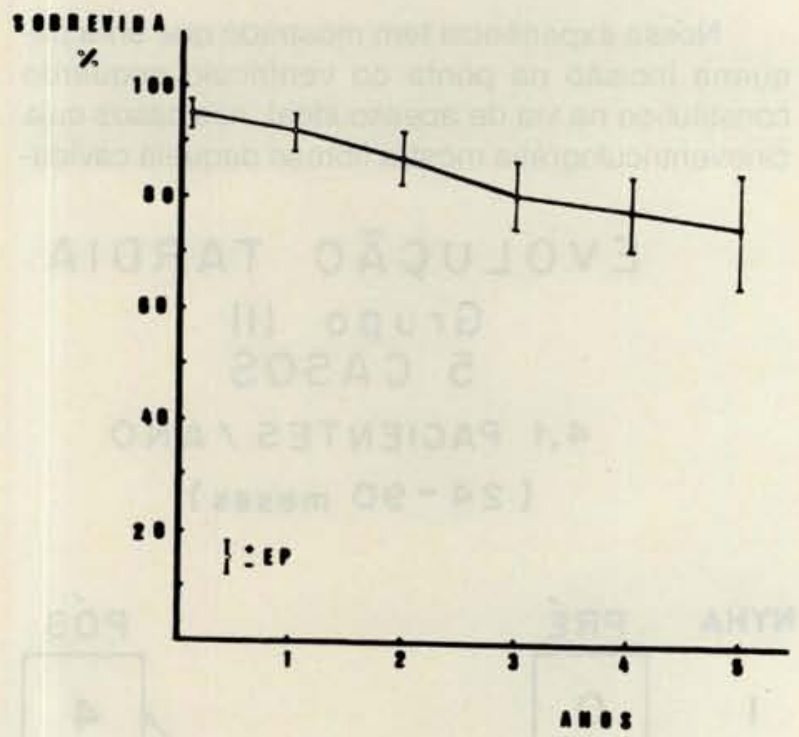

Os resultados do tratamento cirúrgico da endomiocardiofibrose têm sido difíceis de analisar. Uma revisão da literatura (Tabela 12) mostra que, excetuando nossa casuística previamente relatada, existem 81 casos de endomiocardiofibrose operados e publicados. Entretanto, a maioria das publicações refere-se à descrição de casos isolados.

Parece-nos óbvio que a cirurgia da endomiocardiofibrose resulte em uma elevada mortalidade imediata. Em nossa experiência, ela alcançou $20,7 \%$. comparável, portanto, à mortalidade obtida por DUBOST et alii ${ }^{14}(15 \%)$ e por MÉTRAS et alii ${ }^{25}$ (20\%). Para a mortalidade, contribuíram tanto causas cardíacas como extracardíacas. Tentamos identificar possiveis diferenças entre os pacientes com doença bilateral e aqueles com lesão em apenas um ventrículo, pois nos pareceu lógico que os primeiros teriam um risco mais elevado. Os qua- tro óbitos da série relatada por MÉTRAS et alii ${ }^{25}$, referem-se a pacientes com doença bilateral. Em nosso material, a mortalidade foi de $28 \%$, nos casos com lesão biventricular, e de $17 \%$, nos casos com lesão isolada do ventrículo direito. Não houve óbitos, entre os 5 doentes com lesão do ventrículo esquerdo, mas, provavelmente, o pequeno número de casos desse grupo impediu que se obtivesse significação estatística para a impressão clínica de que esses doentes têm um risco operatório menor.

Um outro fato importante na cirurgia da endomiocardiofibrose é o curso tormentoso apresentado pelos doentes, no pós-operatório imediato. Em outras séries ${ }^{14,25}$, a freqüência de complicaçōes tem sido elevada. Em nossa experiência, 50\% dos pacientes que sobreviveram apresentaram complicaçōes (Tabela 6), o que, somado aos 11 doentes falecidos, evidencia uma incidência de complicações fatais e não fatais da ordem de $60 \%$. A análise estatística de todas as complicações, ocorridas no pós-operatório imediato, mostrou serem as complicaçōes cardíacas mais freqüentes (Tabelas 6 e 8), não havendo diferença significativa entre a incidência nos três grupos (Tabela 7).

A mortalidade tardia ocorreu em 10 pacientes $(18,8 \%)$, concorrendo, igualmente, para a mesma, além da insuficiência cardíaca, ou problemas relacionados com a prótese (Tabela 9), causas extracardiacas, e essa cifra é comparável à descrita por DUBOST et alii ${ }^{14}$, que foi de $20 \%$. A relação entre a mortalidade tardia e os diversos grupos não foi significante.

Apenas uma paciente foi reoperada para substituição da valva mitral, que havia sido preservada na primeira intervenção, realizada dois anos antes. Neste caso e numa outra paciente falecida no 6 . mês de pós-operatório e submetida à necropsia, não se observou recidiva da fibrose endocárdica. Esta é uma questão que tem sido freqüentemente levantada e fonte de controvérsias ${ }^{2}{ }^{21}$. É possivel que recidiva da fibrose possa ocorrer após a operação, mas, até o presente, nenhum caso foi relatado.

A curva atuarial mostrou que a probabilidade de sobrevida, decorridos 5 anos da cirurgia, é de $75 \%$ e esta cifra pode ser considerada satisfatória, quando comparada à de outras cardiopatias operadas. Mais importante, contudo, em nossa opinião, é a situação clínica ao longo prazo dos doentes operados. A evolução atual dos 32 sobreviventes (Figura 1 ) revela que 22 , ou seja, somente $41,5 \%$ dos casos operados, estão assintomáticos (classe funcional I ou II da New York Heart Association). O período de evolução é de 4,1 pacientes/ano, va- 
TABELA 12

EXPERIENCIA CIRÚRGICA RELATADA NA ENDOMIOCARDIOFIBROSE

\begin{tabular}{|c|c|c|c|c|c|c|}
\hline \multirow{2}{*}{ N: REFERÉNCIA } & \multirow{2}{*}{ N: CASOS } & \multicolumn{3}{|c|}{ LOCALIZAÇAOAO } & \multicolumn{2}{|c|}{ OBITOS } \\
\hline & & $B V$ & $V D$ & $V E$ & IMEDIATOS & TARDIOS \\
\hline 3 & 1 & - & - & 1 & - & - \\
\hline \multicolumn{7}{|c|}{ 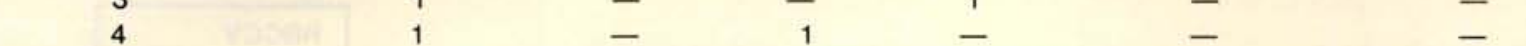 } \\
\hline \multicolumn{7}{|c|}{-} \\
\hline \multicolumn{7}{|c|}{ - } \\
\hline 8 & 2 & 1 & - & 1 & - & - \\
\hline \multicolumn{7}{|l|}{14} \\
\hline \multicolumn{7}{|l|}{15} \\
\hline \multicolumn{7}{|l|}{16} \\
\hline \multicolumn{7}{|c|}{ 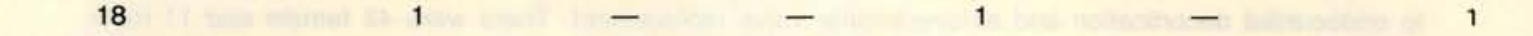 } \\
\hline \multicolumn{7}{|c|}{1} \\
\hline \multicolumn{7}{|l|}{20} \\
\hline \multicolumn{7}{|c|}{ 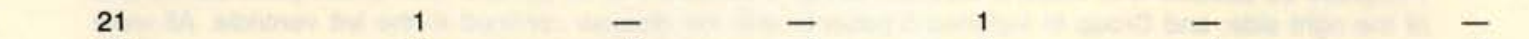 } \\
\hline 23 & 15 & 9 & 4 & 2 & 1 & 1 \\
\hline $\begin{array}{ll}23 & 15 \\
26 & 20\end{array}$ & 20 & 7 & 7 & 6 & 4 & 1 \\
\hline \multicolumn{7}{|c|}{$x_{2}$} \\
\hline \multicolumn{7}{|c|}{ 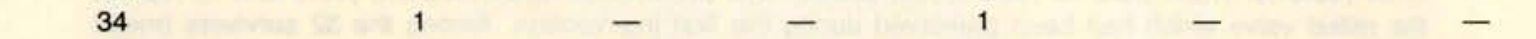 } \\
\hline \multicolumn{7}{|l|}{35} \\
\hline 37 & 10 & 2 & - & - & - & - \\
\hline \multirow[t]{2}{*}{38} & 1 & - & - & 1 & - & - \\
\hline & 81 & 31 & 23 & 27 & 8 & 8 \\
\hline
\end{tabular}

$\mathrm{BV}=$ biventricular $\quad \mathrm{VD}=$ ventrículo direito

$\mathrm{VE}=$ ventriculo esquerdo

riando de 2 a 96 meses. Tal como foi observado por MÉTRAS et alii ${ }^{25}$, a evolução tardia dos pacientes com lesão do lado esquerdo (Figura 4) é melhor do que a evolução dos casos com doença bilateral ou ventricular direita isolada (Figuras 2 e 3 ). Deste modo, 10 pacientes dos grupos I e II apresentam manifestações importantes de insuficiência cardíaca. Provavelmente, eles foram operados numa fase na qual havia perda da força contrátil do ventrículo direito, seja por comprometimento miocárdico decorrente da extensão da fibrose, seja por uma grande e irreversível dilatação das cavidades direitas. Ademais, pacientes com longa evolução da enfermidade apresentam lesão hepática irreversível e alguns deles continuam apresentando ascite, apesar da evidência de boa função cardíaca. Estes fatos nos têm levado a pensar em modificar nossa conduta, passando a indicar precocemente a cirurgia, em todos os casos de endomiocardiofibrose.

\section{CONCLUSÕES}

1) A endomiocardiofibrose é uma doença passível de correção cirúrgica através da endocardiectomia associada à substituição, ou a reparo, das valvas atrioventriculares.
2) A endocardiectomia direita é, sempre, uma operação fácil, se bem que, freqüentemente, produza arritmias, especialmente bloqueio $\mathrm{A}-\mathrm{V}$ total.

3) A endocardiectomia esquerda torna-se difícil, quando o átrio esquerdo é de tamanho normal. Nesta situação, o acesso ao ventrículo esquerdo pode ser realizado através do septo interatrial, ou por ventriculotomia esquerda.

4) Nos casos que apresentam fibrose do ventrículo esquerdo e valva mitral competente, ou com pequena regurgitação, a via de acesso ideal para a endocardiectomia é uma pequena ventriculotomia esquerda.

5) Em virtude do reduzido tamanho da cavidade ventricular na endomiocardiofibrose, é aconselhável o emprego de prótese de baixo perfil.

6) O tratamento cirúrgico da endomiocardiofibrose resulta em elevada mortalidade imediata e tardia.

7) O curso pós-operatório imediato é tormentoso, na maioria dos doentes, e as complicações cardíacas são mais freqüentes.

8) A curva atuarial mostra que a probabilidade de sobrevida, aos 5 anos após a cirurgia, é de $75 \%$. 
MORAES, C. R.; RODRIGUES, J. V.: GOMES, C. A.; MARINUCCI, L.: COELHO. T. C.: SANTOS. C. L.: VICTOR, E.; CAVALCANTI,

I. - Dez anos de cirurgia da endomiocardiofibrose: o que aprendemos? Rev. Bras. Cir. Cardiovasc., 2(1):42-52, 1987.

9) A evolução clínica, a longo prazo, é favorável, em cerca de $40 \%$ dos casos operados.

10) No momento, o tratamento cirúrgico repre- senta a única esperança de modificação no grave prognóstico da endomiocardiofibrose e é provável que melhores resultados imediatos e tardios sejam obtidos, se a operação for indicada precocemente.

RBCCV

MORAES, C. R.; RODRIGUES, J. V.; GOMES, C. A.; MARINUCCI, L.; COELHO, T. C.; SANTOS, C. L.; VICTOR, E.; CAVALCANTI, I. - Ten years of surgery for endomyocardial fibrosis: what have we learned? Rev. Bras. Cir. Cardiovasc., 2(1):42-52, 1987

ABSTRACT: In the last 10 years(1977-1987), 53 patients with endomyocardial fibrosis were submitted to endocardial decortication and atrioventricular valve replacement. There were 42 female and 11 male patients, ranging in age from 11 to 59 years (mean 31). The patients were divided into three groups: Group I included 25 patients with bilateral disease; Group II consisted of 23 patients with endomyocardial fibrosis of the right side; and Group III included 5 patients with the disease confined to the left ventricle. All were in Functional Class III or IV (New York Heart Association classification). The 30-day mortality was $20.7 \%$ (11 cases). The remainder 42 patients survived the early postoperative period which was quite stormy in $21(39.6 \%)$. There were $10(18.8 \%)$ late deaths. One patient was reoperated two years later to replace the mitral valve which had been preserved during the first intervention. Among the 32 survivors (mean follow-up 4.1 patients/year), $22(41,5 \%)$ are in Functional Class I or II. The actuarial curve showed that the 5 -year probability of survival is $75 \%$. Despite the high early and late mortality, and the fact that only $41.5 \%$ of the operated patients had good clinical improvement in the late postoperative period, surgical treatment is, at present, the only hope for patients with endomyocardial fibrosis. Technical aspects of the operation are outlined.

DESCRIPTORS: endomyocardial fibrosis, surgery.

\section{REFERÊNCIAS BIBLIOGRÁFICAS}

1 ARMITAGE, P. - Statistical methods in medical research. Oxford, Blackwell Scient. Publ., 1977. 504 p.

2 BERTRAND, E.; CHAUVET, J.O.D.; ASSAMOI, M.; N'DORI, R.; EKRA, A.; RAVINET, L.; LONGECHAUD, A.; MÉTRAS, C. - Evaluation des résultats du traitement chirurgical de la fibrose endomyocardique: étude de 31 malades opérées et 30 malades non opérées. Bull. Acad. Natt. Méd., 166 (8): 1170-1186, 1982.

3 BINET, J. P.; PERNOD, J.; KERMAREC, J.; COLETTE, J.; WEILER, M.; BOUHEY, J.; BOUVIER, M. - Endocardite constrictive fibroplastique: a propos d'une forme localisée au ventric gauche. Arch. Mal. Coeur, 70 (2): 163-168, 1977.

4 BJORK, V. O.; SZAMOSI, A.; TORNELL, G. - Parietal fibroplastic endocarditis (Loeffler's disease): radiological and surgical aspects in connection with a case report. Scand. J. Thorac. Cardiovasc. Surg., 8 (1): 23-26, 1974.

5 BLAKE, D. P.; PALMER, T. E.; OLINGER, G. N. - Mitral valve replacement in idiopathic hypereosinophilic syndrome. J. Thorac. Cardiovasc. Surg., 89 (4): 630-638, 1985.

6 CACHERA, J.P.; POULAIN, H.; MENASKE, P.; LAURENT, F.; LOISANCE, D.; CHICHE, P. - Endocardite fibreuse d'origine filarienne: traitement chirurgical. Trop. Cardiol. 2: 79, 1976.
7 CORDEIRO, G. M. - Modelos lineares generalizados, Campinas, Editora Universidade Estadual de Campinas, $1986.286 p$.

8 DAVIES, J.; SAPSFORD, R.; BROOKSKY, I.; OLSEN, B. G. J.; SPRY, C. J. F.; OAKLEY, C. M.; GOODWIN, J. F. - Successful surgical treatment of two patients with eosinophilic endomyocardial disease. Br. Heart J., 46 (4) : 438-445, 1981.

9 DUBOST, C. - L'endocardectomie: traitement chirurgical de la fibrose endocardique constrictive. C. A. Acad. Sc., 281: 855-857, 1975.

10 DUBOST, C. - Endocardite fibroplastique de Loefler et fibrose endomyocardique de Davies: traitement chirurgical. Bull. Acad. Natl. Med., 162 (6): 487-493, 1978.

11 DUBOST, C. - Surgery for constrictive fibrous endocarditis. Cardiovasc. Dis., 5: 28, 1979.

12 DUBOST, C. - The surgical traitement of constrictive fibrous endocarditis. J. Cardiovasc. Surg., 19 (6): 581-584, 1978.

13 DUBOST, C.; MAURICE, P.; GERBAUX, A.; BERTRAND, E.; RULLIERE, R.; VIAL, F.; BARILLON, A.; PRIGENT, C.; CARPENTIER, A.; SOYER, R. - The surgical treatment of constrictive fibrous endocarditis. Ann. Surg., 184 (3): 303-307, 1976. 
MORAES, C. R.; RODRIGUES, J. V.; GOMES, C. A.; MARINUCCI, L.: COELHO. T. C.: SANTOS, C. L.: VICTOR. E.: CAVALCANTI.

I. - Dez anos de cirurgia da endomiocardiofibrose: o que aprendemos? Rev. Bras. Cir. Cardiovasc.. 2(1):42-52, 1987.

14. DUBOST, C.; PRIGENT, C.; GERBAUX, A.; MAURICE, P.; PASSELECQ, J.; RULLIERE, R.; CARPENTIER, A.; DELOCHE, A. - Surgical treatment of constrictive fibrous endocarditis. J. Thorac. Cardiovasc. Surg., 82 (4): 585-591, 1981.

15 GRAHAM, J. M.; LAWRIE, G. M.; FETEIH, N. M.; DeBAKEY, M. E. - Management of endomyocardial fibrosis: successful surgical treatment of biventricular involvement and considerations of the superiority of operative intervention. Am. Heart J., 102 (4): 771-782, 1981.

16 HESS, O. M.; TURINA, M.; SENNING, A.; GOEBEL, N. H.; SCHOLER, Y.; KRAYENBUCHL, H. P. - Pre-and postoperative findings in patients with endomyocardial fibrosis. Br. Heart J., 40 (4) : 406-415, 1978.

17 HILL, A. S. - A shorttextbook of medical statistics. London. Hodder and Stoughton, 1978. 325 p.

18 IKÄHELMO, M. J.; KÅRKÖLA, P. J.; TAKKUNEN, J. T. - Surgical treatment of Loeffler's eosinophilic endocarditis. Br. Heart J., 45 (6): 729-732, 1981.

19 JOHN, S.; MANI, G. K.; MURALIDHARAN, S.; KRISHNASWAMY, S.; CHERIAN, G. - Endomyocardial fibrosis from a surgical standpoint $J$. Thorac. Cardio vasc. Surg. 80 (3): 437-440, 1980

20 LAING, H. C.; SHARRATT, G. P.; JOHNSON, A. M.; DAVIES, M. J.; MONRO, J. L. - Endomyocardial fibrosis in an European woman and its successful surgical treatment. J. Thorac. Cardiovasc. Surg., 74 (5): 803-807, 1977.

21 LEPLEY Jr., D.; ARIS, A.; KORNS, M. E.; WALKER, J. A.; D'CUNHA, R. M. - Endomyocardial fibrosis: a surgical approach. Ann. Thorac. Surg., 18 (6): 626-633, 1974.

22 LIMA, R. C.; ESCOBAR, M.; RODRIGUES, J. V.; ANDRADE, J. C. S.; BUFFOLO, E.; MORAES, C. R. - Resultados imediatos e tardios da cirurgia da endomiocardiofibrose. Arq. Bras. Cardiol, 38 (4): 259-267, 1981.

23 MENDONÇA, J. T.; CARVALHO, M. R.; FRANCO, E. F.; KAKUDA, R.; BARROS, M. A. L.; COSTA, G. B.; ALMEIDA, M. L. D.; GARCIA, M. H.; TAQUEDA, S. S.; ARAUJO, J.; SOUZA, A.; MENDONÇA, L. R. - Endomiocardiofibrose: tratamento cirúrgico. Arq. Bras. Cardiol., 42 (Supl. 1): 148, 1986.

24 MÉTRAS, D.; COULIBALY, A. Q.; QUATTARA, K.; KANGAH, K.; CHAUVET, J.; N'DORI, R.; BERTRAND, E. - La chirurgie valvulaire conservatrice dans la fibrose endomyocardique: a propos de 8 cas sur 46 opérés. Arch. Mal. Coeur, 77 (7): 806-811, 1984.

25 MÉTRAS, D.; QUEZZIN-COULIBALY, N.; QUATTARA, K.; CHAUVET, J.; EKRA, A.; LONGECHAUD, A.; BERTRAND, E. - Endomyocardial fibrosis: early and late results of surgery in 20 patients. J. Thorac. Cardiovasc. Surg., 83 (1): 52-64, 1982.

26 MÉTRAS, D.; QUEZZIN-COULIBALY, N.; QUATTARA, K.; LONGECHAUD, A.; CHAUVET, J.; BERTRAND, E. Le traitement chirurgical de la fibrose endomyocardique constrictive: a propos de 15 cas. Arch. Mal. Coeur, 74 (8): 939-950, 1981.
27 MORAES, C. R. - Cirurgia da endomiocardiofibrose. Recife, 1980. (Tese Titular - Universidade Federal de Pernambuco).

28 MORAES, C. R.; BUFFOLO, E.; LIMA, R.; VICTOR, E.; LIRA, V.; ESCOBAR, M.; RODRIGUES, J.; SARAIVA, L.; ANDRADE, J. C. - Surgical treatment of endomyocardial fibrosis. J. Thorac. Cardiovasc. Surg., 85 (5): $738-745,1983$

29. MORAES, C. R.; BUFFOLO, E.; VICTOR, E.; SARAIVA, L.; GOMES, J. M. P.; LIRA, V.; LIMA, R.; ESCOBAR, M.; ANDRADE, J. C. S. - Endomyocardial fibrosis: report of 6 patients and review of the surgical literature. Ann. Thorac. Surg., 29 (3): 243-248, 1980.

30 MORAES, C. R.; ESCOBAR, M.; LIMA, R.; RODRIGUES, J. V. - Technical aspects in surgery for endomyocardial fibrosis: experience with 37 patients. Texas Heart Inst., J., 10 (2): 115-118, 1983.

31 MORAES, C. R.; VICTOR, E. G.; SARAIVA, L.; LIRA, V.; GOMES, J. M. P.: ESCOBAR, M.; LIMA, R. C. - Tratamento cirúrgico da endomiocardiofibrose. Arq. Bras. Cardiol., 33 (Supl. 1): 251-257, 1979.

32 NAIR, U.; EVANS, T.; OAKLEY, D. - Surgical treatment of endomyocardial fibrosis with preservation of mitral valve. Br. Heart J., 43 (3): 357-359, 1980.

33 PRIGENT, C.; BACULARD, P.; CARPENTIER, A.; PASSELECQ, J.; DUBOST, C. - Endocardite fibreuse du ventricle gauche chez un filarien, cure chirurgicale. Arch. Mal. Coeur, 66 (9): 1101-1110, 1973.

34 SAN JUAN, E. - Comentários. In: MORAES, C. R. et alii. Tratamento cirúrgico da endomiocardiofibrose. Arq. Bras. Cardiol., 33 (Supl. 1): 257, 1979.

35 SHEIKHZADEH, A. H.; TARBIAT, A.; NAZARIAN, I.; ARYANPUR, I.; SENNING, A. - Constrictive endocarditis: report of a case with successful surgery. $\mathrm{Br}$. Heart J., 42 (2): 224-228, 1979.

36 SIEGEL, S. - Nonparametric statistics for the behavioral sciences. Tokyo, McGraw Hill Book Company, Inc., 1976. 350 p.

37 STOLF, N. A. G.; COSTA, R.; PILLEGI, F.; MACRUZ, R.; VERGINELLI, G.; BITTENCOURT, D.; ZERBINI, E. J. - Tratamento cirúrgico da endomiocardiofibrose: relato de um caso e revisāo da literatura. Arq. Bras. Cardiol., 33 (2): 125-131, 1979.

38 WOOD, A. E.; BOYLE, D.; O'HARA, M. D.; CLELAND, J. - Mitral annuloplasty in endomyocardial fibrosis: an alternative to valve replacement. Ann. Thorac. Surg., 34 (4): 446-451, 1982.

\section{Discussão}

DR. JOSÉ TELES DE MENDONÇA Aracajú, SE

Gostaria de cumprimentar o Prof. Carlos Moraes pela pela excelência do trabalho apresentado, 
ressaltando a importância do que nos foi ensinado por ele, ao longo dos últimos dez anos. Iniciamos nossa experiência com o tratamento cirúrgico dessa patologia em março de 1982 e já tivemos a oportunidade de operar 15 pacientes. Nove tinham a forma biventricular, 4 do ventrículo direito e 2 do ventrículo esquerdo. A cirurgia realizada consistiu na endocardiectomia ventricular mais substituição das valvas atrioventriculares comprometidas. Houve um único óbito $(6,6 \%)$ no pós-operatório imediato e dois no pós-operatório tardio. Quatro pacientes necessitaram de marcapasso permanente, sendo que 2 já tinham indicação antes da cirurgia (1 BAVT intermitente em 1 fibrilação atrial, com freqüência ventricular lenta. Dois desenvolveram BAVT durante 0 ato cirúrgico. Dos sobreviventes, 10 apresentaram uma boa evolução e 2 evoluem apenas satisfatoriamente. Utilizando o espírito imposto pelo autor, gostaria de comentar alguns pontos que julgamos importantes, no manuseio da endomiocardiofibrose. 1) A utilização sistemática do ecocardiograma bidimensional poderá favorecer o diagnóstico em um número maior de pacientes (áreas endêmicas), inclusive o diagnóstico na fase aguda (ultrassom colorido-Davies), bem como será de extrema valia no acompanhamento dos pacientes operados. 2) A cirurgia deve ser precoce, para evitar as lesões extracardíacas (hepáticas e pulmonares), mas, sobretudo, para evitar a progressão das lesões miocárdicas (atrofia e fibrose). 3) Deve-se evitar o acesso transventricular, preservando, assim, maior número possível de fibras musculares. 4) A cavidade decorticada permanece pequena no pós-operatório imediato e a válvula implantada deve, por esta razão, ser, obrigatoriamente, de baixo perfil. 5) A técnica preconizada por Metras contribuiu, decisivamente, para diminuir a incidência de bloqueios cirúrgicos. 6) A cirurgia é um método efetivo de tratamento; pode ser realizada com mortalidade relativamente baixa e muda favoravelmente a história natural dessa terrivel doença.

\section{DR. NILZO RIBEIRO}

Salvador, $B A$

Inicialmente, os meus cumprimentos ao $\mathrm{Dr}$. Carlos Moraes e colaboradores, pelo brilhante trabalho apresentado. Quanto à nossa experiência, ela tem mostrado que existe um plano de clivagem nítido entre a fibrose e o miocárdio sadio. Deste modo, uma dissecção delicada permite uma ressecção perfeita desta carapaça, pois a fibrose é como um tecido aposto ao miocárdio. Qual a opinião do Dr. Moraes?

\section{DR. MORAES \\ (Encerrando)}

Inicialmente, gostaria de agradecer as palavras elogiosas dos comentadores. Eu concordo com todas as colocações do Dr. Teles, exceto que a via transventricular deva ser evitada. Continuo pensando que $o$ acesso transventricular é útil em pacientes com fibrose do VE e discreta insuficiência mitral. Também concordo, inteiramente, com o Dr. Nilzo em que existe um plano de clivagem bem nítido entre a fibrose e o miocárdio subjacente, possibilitando uma decorticação perfeita. Para finalizar, gostaria de reiterar a minha filosofia em relação à cirurgia da EMF: trata-se de uma doença grave, a cirurgia implica em risco considerável e a avaliação tardia dos doentes operados é imprescindivel, para que se possa tirar conclusōes mais definitivas sobre esse tipo de procedimento. 